\title{
HarpyGame: A Customizable Serious Game for Upper Limb Rehabilitation after Stroke
}

\author{
Gabriel Cyrino \\ Faculty of Electrical Engineering \\ Federal University of Uberlândia \\ Uberlândia, Brazil \\ gabrielcyrino@ufu.br
}

\author{
Júlia Tannús \\ Faculty of Electrical Engineering \\ Federal University of Uberlândia \\ Uberlândia, Brazil \\ julia.tannus95@gmail.com
}

\author{
Edgard Lamounier \\ Faculty of Electrical Engineering \\ Federal University of Uberlândia \\ Uberlândia, Brazil \\ lamounier@ufu.br
}

\author{
Alexandre Cardoso \\ Faculty of Electrical Engineering \\ Federal University of Uberlândia \\ Uberlândia, Brazil \\ alexandre@ufu.br
}

\author{
Alcimar Soares \\ Faculty of Biomedical Engineering \\ Federal University of Uberlândia \\ Uberlândia, Brazil \\ alcimar@ufu.br
}

\begin{abstract}
Summary-Stroke is the most common disease that leads to the dexterity impairment of the upper limbs. Serious games have emerged as an advantageous and enjoyable alternative to rehabilitation treatment when compared to traditional therapies. This work presents the development of a customizable serious game, based on Virtual Reality techniques to achieve a more natural and intuitive interface. The game was developed to allow different types of input devices. In so doing, the system consists of a control panel for patient management and customization of the game, a serious game with a realistic environment with levels and adaptive tasks, and a database. The research considered the assistance of physiotherapists to provide protocols that the game followed. Results indicated significant acceptance by the patients, implying the system's potential use in the post-stroke rehabilitation process of the upper limbs.
\end{abstract}

Index Terms-Stroke, Virtual Reality, Serious Game, Motor Rehabilitation.

\section{INTRODUCTION}

Stroke is a serious and very common disease. According to the World Health Organization, stroke is the second leading cause of death in the world and occurs predominantly in middle-aged and elderly adults [1].

The most common forms of post-stroke therapy programs consist of the patient to perform repetitive movements under the supervision of a therapist in several individual sessions [2]. However, these traditional approaches tend to be more tedious and less intuitive and may delay or hinder the rehabilitation process, and cause frustration in the patient [3].

An increasingly promising alternative for this purpose is serious games and Virtual Reality (VR) [4]. A serious game goes beyond pure entertainment like video games, as it offers other types of experiences that can be directed toward relearning, rehabilitating, and training individuals [5]. And VR stands out as an advanced interface for applications aimed at human rehabilitation [6].

Some studies have reported benefits of using VR and serious games in rehabilitation, such as better performance of motor skills post-stroke [7], improvement of cognitive functions, neuroplasticity stimulation, and greater autonomy in the daily life activities [8]. Thus, by decreasing the monotony of hundreds of repeated movements and providing performance feedback, games can increase both the quality and quantity of post-stroke home therapy [9].

\section{OBJECTIVE}

This work aims to develop a virtual training system composed of a serious game with a realistic scenario, database integration, and feature customization to meet individual needs, aspects rarely found in searched applications. In addition, it is intended to assess the suitability and acceptance of the system developed as a support tool in post-stroke rehabilitation processes and to assist therapists in defining more specific rehabilitation protocols for each patient.

\section{MAterials AND MethodS}

The serious game consists of a 3D environment where the patient controls a harpy eagle, a typical Brazilian bird, using the positioning of his arm. The scenario consists of a virtual forest containing a series of objectives and challenges, such as avoiding obstacles and catching food.

The game was developed in Unreal Engine 4. In this game engine, the 3D virtual environment was put together and scripts with the game logic were programmed. Game models and animations were made using Blender 3D, an open-source creation suite.

The patient's forearm movements are tracked by the input device connected to the game. The system was designed mainly to communicate with the Myo device accelerometer, a human-computer interaction bracelet composed of several sensors. The game also provides the option of being controlled by conventional joysticks, and can also be configured for other input devices, such as a proprietary assisted platform. The 
movement of the harpy is made based on the data from these devices.

Thus, the patient's limb displacement can be measured, allowing the motor and functional rehabilitation to be monitored. As a result, to enable the main character movement, the patient ends up doing exercises: internal and external rotation of the arm enables movement in the right-left axis, while elbow flexion and extension moves the character in the up-down axis.

Initially, it was decided to develop four stages in the game. Each level has a different goal and works a specific gesture, namely:

- Interactive Tutorial: "Follow the arrows"-The patient should follow the direction of some arrows. In this training, arm extension in four directions is trained.

- Level 1: "Pass through the rings"-The patient should pass inside several rings. It is necessary to make gentle and firm movements with the arm.

- Level 2: "Catch 5 fish"-The patient should catch 5 fish in the lake. The catch must be quick and precise, working out rapid elbow flexion and extension.

- Level 3: "Hunt 5 meats"-The patient must capture 5 pieces of meat, while running away from predators. This phase works general coordination and speed.

The system also consists of a control panel, where the therapist can register, change and consult patient data, configure the general game parameters, select the game levels in each session and configure the challenges for those selected levels. Therefore, it is possible to create a custom execution protocol, configuring the sessions based on the therapeutic needs of each patient.

The data relating to the settings made by the therapist and the actions of the patient during the sessions are stored in a common database that was modeled using the SQLite library.

After a formal authorization by the Federal University of Uberlândia ethics committee, a user study was conducted with 6 post-stroke volunteer patients, aged between 35 and 73. They were selected by the therapist according to some exclusion criteria. For each patient, 6 sessions of 30 minutes each were made, two per week, always accompanied by a developer and a therapist. A training protocol was defined where the difficulty of the game is gradually increased or decreased by the therapist, without discouraging the patient.

The evaluation methods involved quantitative and qualitative analyzes. The quantitative analysis was based on the training protocol, evaluating the percentage of challenges overcome during the game, the modified parameters and the time spent to complete the levels.

For the qualitative analyzes, after all sessions, the users answered some questions about their experience using the game. The first questionnaire applied was the System Usability Scale (SUS). It is a general usability scale developed by John Brooke [10]. Another questionnaire was also applied, with 8 specific questions regarding game appeal, relevance, immersion, motivation, and perceived user skill improvement.

\section{RESUlTS AND Discussion}

From the analysis of the challenges overcome the time to complete the levels, a significant evolution of patients was observed. However, this evolution has not remained stable. This is because the parameters are made difficult or facilitated according to the patients' adaptation and learning.

Overall, the game was well accepted by the patients. The result of the SUS questionnaire shows that the system fulfills the criteria of effectiveness, efficiency, and satisfaction, according to the scale. Also, our calculated SUS score was 85.5 out of 100 points, indicating that the system has good usability.

The results from the additional questionnaire indicate that the patients found the use of HarpyGame relevant, engaging, and suitably challenging. They felt that they were making good progress while playing and that their skill improved above average. The proposed activities were neither too easy nor too difficult, promoting the challenges at an appropriate pace.

\section{CONCLusions}

Through the results obtained, it can be concluded that the developed system has the potential to be used in the rehabilitation of patients with paresis in the upper limbs resulting from a stroke. Compared to conventional therapy, this VR alternative allows adapting the exercises to the patients' interests and habits, potentially increasing their motivation. The application's playful design motivated patients and caregivers to continue with the treatment.

\section{ACKNOWLEDGMENT}

The autors acknowledge the CAPES Institute (Coordenação de Aperfeiçoamento de Pessoal de Nível Superior) for the support of the project.

\section{REFERENCES}

[1] Organização Mundial de Saúde, "Acidente vascular cerebral (avc)," http://www.brasil.gov.br/editoria/saude/2012/04/acidente-vascularcerebral-avc, Dec. 2017, (Acesso em: 15-Junho-2018).

[2] P. Langhorne, F. Coupar, and A. Pollock, "Motor recovery after stroke: a systematic review," The Lancet Neurology, vol. 8, no. 8, pp. 741-754, 2009.

[3] M. F. Levin, P. L. Weiss, and E. A. Keshner, "Emergence of virtual reality as a tool for upper limb rehabilitation: Incorporation of motor control and motor learning principles," Physical Therapy, vol. 95, no. 3, pp. 415-425, sep 2014.

[4] M. Ma, A. Oikonomou, and L. C. Jain, Serious games and edutainment applications. Springer, 2011, vol. 504.

[5] D. R. Michael and S. L. Chen, Serious games: Games that educate, train, and inform. Muska \& Lipman/Premier-Trade, 2005.

[6] R. Tori, C. Kirner, and R. A. Siscoutto, Fundamentos e tecnologia de realidade virtual e aumentada. Editora SBC, 2006.

[7] G. Saposnik and M. Levin, "Virtual reality in stroke rehabilitation a meta-analysis and implications for clinicians," Stroke; a journal of cerebral circulation, vol. 42, pp. 1380-6, 052011.

[8] L. B. G. S. D. J. S. G. Laver, KE and M. Crotty, "Virtual reality for stroke rehabilitation," Cochrane Database of Systematic Reviews, no. 11, 2017.

[9] G. Alankuş, A. Lazar, M. May, and C. Kelleher, "Towards customizable games for stroke rehabilitation," in Proceedings of the twenty-eighth ACM SIGCHI conference on computer human interaction (CHI), vol. 3, 01 2010, pp. 2113-2122.

[10] J. Brooke et al., "Sus-a quick and dirty usability scale," Usability evaluation in industry, vol. 189, no. 194, pp. 4-7, 1996. 